\title{
Luteinization of Ovarian Transplants in Gonadectomized Male and Female Rats under Stressful Conditions and Its Relation to Sexual Differentiation of the Hypothalamus
}

\author{
TAKEO MACHIDA \\ Zoological Institute, Faculty of Science, University of Tokyo, Tokyo
}

\begin{abstract}
Synopsis
In the 1st series of experiments, male and female rats were gonadectomized at 10 days of age and simultaneously given subcutaneous syngenesio- or autotransplants of an ovary. At 25 days of age a group of the rats were subjected to stressful stimuli by making a subcutaneous croton oil pouch. Another group of the rats not exposed to stress served as controls. When they were sacrificed at 35 days of age, corpora lutea (CL) were never found in the ovarian grafts in both the control and stressed female rats. On the contrary, in 7 out of 12 stressed male hosts the grafts contained well developed CL, although those in the control animals invariably lacked them. In the 2nd series of experiments, male rats were orchidectomized on the day after birth, all the other procedures being similar to those in the preceding series. At sacrifice, CL were encountered in the ovarian grafts in 6 out of 11 control rats. However, in 10 similar rats exposed to stressful stimuli, CL were formed in only one animal. These and the writer's previous findings seem to indicate that an increase in the release of GTH, LH in particular, following exposure to stressful stimuli is in some way associated with the male pattern of GTH secretion.
\end{abstract}

In animals exposed to stressful stimuli the gonad is reduced in activity, probably because of a decrease in hypophyseal gonadotropin secretion, while the adrenal cortex undergoes a hypertrophy as a result of an increased secretion of ACTH from the hypophysis (Selye, 1946; Baker, 1952).

On the other hand, continued exposure of neonatally estrogenized persistent-estrous rats to stress results in luteinization in otherwise anovulatory polyfollicular ovaries of the animals, suggesting an increased release of gonadotropins (GTH), especially $\mathrm{LH}$, from the anterior hypophysis (Takasugi, 1956a, b). It is well established that in such persistentestrous rats GTH are secreted at a constant rate, as in the case of male rats (Takasugi, 1952; Takewaki, 1962).

Luteinization also frequently occurs in

Received for publication May 21, 1970. subcutaneous ovarian transplants in castrated male rats, if the hosts are subjected to stressful stimuli by making a subcutaneous croton oil pouch (Takewaki, 1956; Machida, 1968a, b). Subcutaneous ovarian grafts in orchidectomized rats normally develop follicles but seldom form corpora lutea (Sand, 1919; Takewaki, 1933; Takasugi, 1952).

In immature rats, luteinization of the ovaries following injections of PMS or estrogen is greatly suppressed under stressful croton oil treatment, probably as a result of inhibition of an increase in LH release from the anterior hypophysis which would take place after the injection of PMS or estrogen (Machida, 1969).

A question arises here as to why stressful stimuli could promote the release of $\mathrm{LH}$ in orchidectomized males and neonatally estrogenized persistent-estrous females but not in immature female animals. The aim of the present study was to examine whether luteini- 
zation could occur in ovaries engrafted to gonadectomized male and female rats if the hosts were placed under stressful conditions, and to discuss the possible mechanism involved in the release of $\mathrm{LH}$ following exposure to stressful stimuli.

\section{Materials and Methods}

Male and female rats of the Sprague-Dawley strain from the colony maintained in this laboratory under controlled temperature and lighting (14 hr/day) were used in these experiments.

In the first series of experiments, 23 female rats of 10 days old were spayed and given autotransplants of an ovary under the abdominal skin in single stage operations under ether anesthesia. In the second series of experiments, 41 male rats were divided into two groups on the day of their birth (day 0 of age). Orchidectomies were performed on 21 rats on the day after birth under cold anesthesia and on the remaining 20 animals at 10 days of age under ether anesthesia. At the age of 10 days, all the rats were given subcutaneous transplants of an ovary from a litter-sister. After being weaned at 21 days of age, the rats were kept on rat chow and drinking water ad libitum. When they reached 25 days of age, each rat was given an injection of $4 \mathrm{ml}$ of air under the back skin followed by an injection of $0.4 \mathrm{~m} l$ of $50 \%$ croton oil into the air pouch. Croton oil was diluted with sesame oil. Those bearing ovarian grafts but not given croton oil served as controls.

At autopsy performed at 35 days of age, ovarian grafts, uteri, adrenals and hypophyses were dissected out, weighed and fixed in Bouin's solution. Sections were cut in paraffin at $8 \mu$ and stained with
Delafield's hematoxylin and eosin. Corpora lutea in the ovarian grafts, if they were present, were counted and their diameters measured according to the technique described in the previous paper (Machida, 1969).

\section{Results}

Failure of luteinization in ovarian grafts in immature female rats under stressful conditions (Groups 1-2)

When sacrificed at 35 days of age, fully formed corpora lutea were never encountered in ovarian grafts in rats which had been spayed and given ovarian transplants at 10 days of age (Group 1 in Table 1). In similar females exposed to stressful stimuli(croton oil), luteinization also failed to occur in ovarian grafts until sacrifice at 35 days of age (Group 2). In the majority of Group 2 rats, the grafts were undergoing involution, being no longer found as such in 6 of 14 animals. However, a few animals of both groups had one or two luteinizing follicles in their grafts and developed the vaginal orifice, although they failed to come into vaginal estrus.

The mean weights of adrenals and hypophysis of Group 2 rats were significantly greater than those of Group 1 animals (adrenals, $0.001<\mathrm{p}<0.01$; hypophysis, $0.02<$ $\mathrm{p}<0.05$ ), while the mean body weight and

Table 1. Luteinization of ovarian grafts in gonadectomized immature rats of both sexes, under stressful conditions

\begin{tabular}{ccccccccccc}
\hline \hline Group Sex $\begin{array}{c}\text { Age at } \\
\text { castra- } \\
\text { tion } \\
\text { (days) }\end{array}$ & Treatment & $\begin{array}{c}\text { No. of } \\
\text { rats }\end{array}$ & $\begin{array}{c}\text { No. of } \\
\text { rats } \\
\text { with } \\
\text { CL }\end{array}$ & $\begin{array}{c}\text { No. of CL } \\
\text { per } \\
\text { rat }\end{array}$ & $\begin{array}{c}\text { Body } C L \\
\text { weight } \\
(\mathrm{g})\end{array}$ & \multicolumn{2}{c}{$\begin{array}{c}\text { Organ weight (mg/100g BW) } \\
\text { ovarian } \\
\text { graft }\end{array}$} & adrenals & hypophysis \\
\hline 1 & F & 10 & control & 9 & 0 & - & $64 \pm 14^{* *}$ & $27.7 \pm 8.5$ & $25.0 \pm 3.5$ & $5.64 \pm 0.25$ \\
2 & F & 10 & stressed & $8(14)^{*}$ & 0 & - & $61 \pm 12$ & $28.1 \pm 11.4$ & $31.6 \pm 3.5$ & $6.28 \pm 0.66$ \\
3 & M & 10 & control & 8 & 0 & - & $58 \pm 15$ & $18.2 \pm 0.7$ & $24.1 \pm 1.9$ & $6.14 \pm 0.44$ \\
4 & M & 10 & stressed & 12 & 7 & $2.3 \pm 1.0$ & $75 \pm 43$ & $16.1 \pm 2.9$ & $30.6 \pm 5.2$ & $6.75 \pm 0.52$ \\
5 & M & 1 & control & 11 & 6 & $6.5 \pm 5.6$ & $94 \pm 16$ & $18.7 \pm 6.1$ & $21.2 \pm 3.3$ & $5.71 \pm 0.91$ \\
6 & M & 1 & stressed & 10 & 1 & 1 & $64 \pm 9$ & $22.5 \pm 6.9$ & $30.9 \pm 6.2$ & $5.92 \pm 0.73$ \\
\hline
\end{tabular}

CL: corpora lutea, F: female, $\quad \mathrm{M}$ : male.

* In this group, ovarian grafts were recovered in 8 of 14 rats, disappearing completely in the remaining 6 .

** mean \pm standard error. 
the mean weight of ovarian grafts were not significantly different between the two groups $(\mathrm{p}>0.05)$.

Luteinization of ovarian grafts in immature rats orchidectomized at day 1 or 10 of age, under stressful conditions (Groups 3-6)

In 35-day-old male rats which had been orchidectomized and engrafted with an ovary at 10 days of age, the grafts contained follicles of varying sizes but no corpora lutea (Group 3, Fig. 1). On the contrary, 7 of 12 similar rats which had been placed under stressful conditions (croton oil) developed 1-4 corpora lutea, measuring $250-1000 \mu$ in diameter, in the grafts (Group 4, Fig. 2). The mean weight of adrenals was significantly greater in Group 4 rats than in Group 3 animals $(0.01<\mathrm{p}$ $<0.02$ ), but the mean body weight and the mean weights of ovaries and hypophysis of the stressed rats did not deviate significantly from those of the controls $(\mathrm{p}>0.05)$.
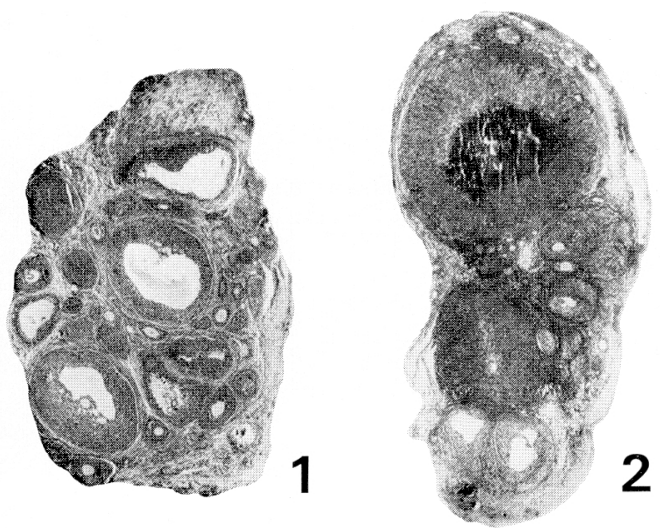

Fig. 1. Ovarian graft recovered from a 35-day-old male rat (Group 3). The host rat was orchidectomized at 10 days of age and at the same time given an ovarian transplant from a litter-sister. The graft contains follicles of varying sizes but no corpora lutea. $\times 30$.

Fig. 2. Ovarian graft recovered from a stressed male rat (Group 4). The host rat, orchidectomized and transplanted with an ovary at 10 days, was placed under stressful conditions of croton oil treatment from 25 days until autopsy. Well-developed corpora lutea are visible. $\times 30$.
Corpora lutea were formed in ovarian grafts in 6 of 11 35-day-old rats which had been orchidectomized at day 1 of age and given transplants at 10 days (Group 5, Fig. 3). However, if similar rats were subjected to stressful stimuli, luteinization of ovarian graft was definitely reduced in incidence, only one of 10 rats developing a corpus luteum of medium size in its graft (Group 6, Fig. 4). Both the body weight and the weight of adrenals of Group 6 rats were significantly different from those of Group 5 ( $p<0.001)$.

In stressed rats (Group 2, 4 and 6), the zona fasciculata of the adrenal cortex was definitely enlarged. In Groups $3-6,10-25 \%$ of male hosts exhibited a few luteinizing follicles in their ovarian grafts.
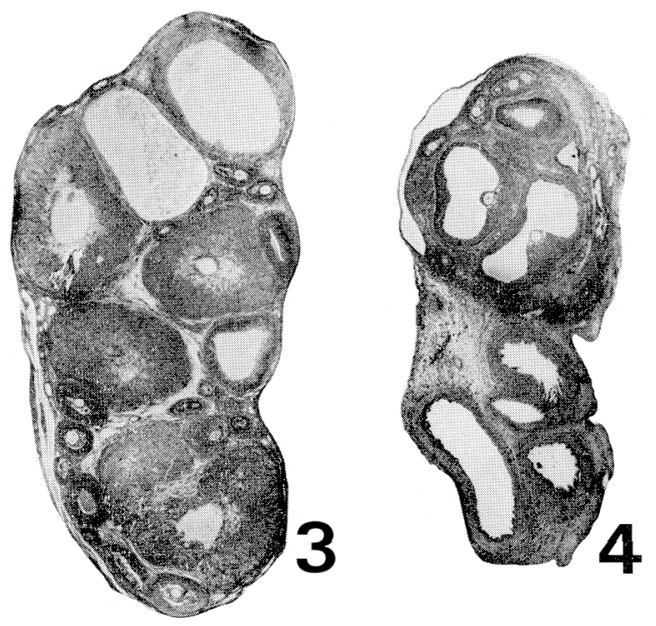

Fig. 3. Ovarian graft recovered from a neonatally orchidectomized male rat (Group 5). Several corpora lutea are visible. $\times 30$.

Fig. 4. Ovarian graft recovered from a neonatally orchidectomized, stressed rat (Group 6). The graft contains follicles but no corpora lutea. $\times 30$.

\section{Discussion}

The ovaries of neonatally estrogenized adult rats showing persistent vaginal estrus (Takasugi, 1956a, b) and subcutaneous ova- 
rian grafts in orchidectomized adult rats (Takewaki, 1956; Machida, 1968a, b) which usually exhibit follicles of varying sizes but lack corpora lutea develop corpora lutea after exposure of the animals to stressful stimuli. In contrast, luteinization of the ovaries of immature rats following PMS or estradiol injections is suppressed under stressful conditions (Machida, 1969). In the present experiment, luteinization took place in subcutaneous ovarian grafts in immature male rats orchidectomized at 10 days (Group 4) following exposure of the hosts to noxious stimuli of croton oil, whereas luteinization failed to occur in similar grafts in immature females ovariectomized at 10 days (Group 2) placed under the same stressful conditions.

Kawashima (1960), Yazaki (1960), Takewaki (1962) and Gorski and Wagner (1965) showed that the tonic pattern of GTH secretion was established in the male rat by the influence on the hypothalamus of androgenic steroids secreted from the testes during the first few days after birth, while the development of the cyclic pattern of GTH discharge in the female rat was independent of normal ovarian activity so that, although the female mode of GTH secretion was established in the male following orchidectomy performed within the first few days after birth, the development of the feminine pattern of GTH secretion in the female was not affected by early postnatal ovariectomy. The pattern once determined is not affected by later gonadectomy in both the male and female rats.

The present findings appear to show that the release of the ovulatory surge of LH from the anterior hypophysis and consequent luteinization of ovarian grafts in response to stressful stimuli are associated with the tonic or male pattern of hypophyseal GTH secretion in the hosts. This conclusion is supported by the results of the last series of experiments showing that in ovarian transplants in male rats orchidectomized on the day after birth, luteinization failed to occur after exposure of the hosts to stress.

When sacrificed at the age of 35 days, corpora lutea were frequently encountered in ovarian grafts in male rats which had been orchidectomized at day 1 of age (Group 5 ), but not at all in neonatally ovariectomized female rats (Group 1). This appears to show that androgens secreted by fetal testes exert an advancing effect on the development of the GTH releasing mechanism in the CNS of the male rat. Zarrow et al. (1969) recently reported that neonatal treatment of female rats with low doses of androgen might induce precocious sexual development and earlier onset of vaginal estrus.

\section{Acknowledgement}

The author is indebted to Dr. N. Takasugi for his valuable advice and encouragement, and to Dr. S. Kawashima for the helpful discussions throughout the present experiments. Cordial thanks are also due to Professor Emeritus K. Takewaki of the University of Tokyo for his criticism and kind help in preparing the manuscript.

\section{References}

Baker, B. L. (1952). Rec. Prog. Horm. Res. 7, 331.

Gorski, R. A. and J. W. Wagner (1965). Endocrinology 76, 226.

Kawashima, S. (1960). J. Fac. Sci., Univ. Tokyo, IV. 9, 117.

Machida, T. (1968a). Ibid. IV. 11, 429.

Machida, T. (1968b). Proc. Japan Acad. 44, 954.

Machida, T. (1969). Annot. Zool. Japon. 42, 68.

Sand, K. (1919). Pflüger's Arch. 173, 1.

Selye, H. (1946). J. Clin. Endocrinol. 6, 117.

Takasugi, N. (1952). Annot. Zool. Japon. 25, 120.

Takasugi, N. (1956a). J. Fac. Sci., Univ. Tokyo, IV. 7, 595.

Takasugi, N. (1956b). Ibid. IV. 7, 605. 
Takewaki, K. (1933). J. Fac. Sci., Imp. Univ. Takewaki, K. (1962). Experientia 18, 1. Tokyo, IV. 3, 155.

Yazaki, I. (1960). Annot. Zool. Japon. 33, 217.

Takewaki, K. (1956). Annot. Zool. Japon. 29, 1. Zarrow, M. X., R. H. Naqvi and V. H. Denenberg (1969). Endocrinology 84, 14. 